\title{
QUOTIENTS OF ÉTALE GROUPOIDS AND THE ABELIANIZATIONS OF GROUPOID C*-ALGEBRAS
}

\author{
FUYUTA KOMURA
}

(Received 15 January 2019; accepted 18 January 2020; first published online 7 April 2020)

Communicated by L. O. Clark

\begin{abstract}
In this paper, we introduce quotients of étale groupoids. Using the notion of quotients, we describe the abelianizations of groupoid $\mathrm{C}^{*}$-algebras. As another application, we obtain a simple proof that effectiveness of an étale groupoid is implied by a Cuntz-Krieger uniqueness theorem for a universal groupoid $\mathrm{C}^{*}$-algebra.
\end{abstract}

2010 Mathematics subject classification: primary 46L05; secondary 22A22.

Keywords and phrases: $\mathrm{C}^{*}$-algebras, étale groupoids.

\section{Introduction}

The study of $\mathrm{C}^{*}$-algebras associated to étale groupoids, groupoid $\mathrm{C}^{*}$-algebras, was initiated by Renault in [10]. Since then, many researchers have studied the relationship between étale groupoids and groupoid $\mathrm{C}^{*}$-algebras. In the previous studies, there are many results for $\mathrm{C}^{*}$-algebras associated to Hausdorff étale groupoids. However, $\mathrm{C}^{*}$ algebras associated to non-Hausdorff étale groupoids do not seem to have been studied sufficiently. Non-Hausdorff groupoids naturally arise as mentioned in [5, 8] and so on. Exel pointed out in [7] that some results known for Hausdorff étale groupoids do not necessarily hold for non-Hausdorff groupoids. In [4], the authors treat simplicity of groupoid $C^{*}$-algebras associated to non-Hausdorff étale groupoids.

In this paper we calculate the abelianization of a groupoid $\mathrm{C}^{*}$-algebra. For a discrete group $\Gamma$, the abelianization $C^{*}(\Gamma)^{\text {ab }}$ of its group $C^{*}$-algebra $C^{*}(\Gamma)$ is isomorphic to $C^{*}\left(\Gamma^{\mathrm{ab}}\right)$, where $\Gamma^{\mathrm{ab}}$ is the abelianization of $\Gamma$. Furthermore, $C^{*}\left(\Gamma^{\mathrm{ab}}\right)$ is isomorphic to $C\left(\widehat{\Gamma^{\mathrm{ab}}}\right)$, where $\widehat{\Gamma^{\mathrm{ab}}}$ is the Pontryagin dual of $\Gamma^{\mathrm{ab}}$. It is natural to consider an étale groupoid analogy. For an étale groupoid $G$, we construct an étale groupoid $G^{\mathrm{ab}}$ and a topological groupoid $\widehat{G^{\mathrm{ab}}}$ so that $C^{*}(G)^{\mathrm{ab}} \simeq C^{*}\left(G^{\mathrm{ab}}\right) \simeq C_{0}\left(\widehat{G^{\mathrm{ab}}}\right)$ holds. In order to

This work was supported by the RIKEN Junior Research Associate Program.

(C) 2020 Australian Mathematical Publishing Association Inc. This is an Open Access article, distributed under the terms of the Creative Commons Attribution licence (http://creativecommons.org/licenses/ by/4.0/), which permits unrestricted re-use, distribution, and reproduction in any medium, provided the original work is properly cited. 
construct $G^{\text {ab }}$, we introduce the notion of quotient étale groupoids. A quotient étale groupoid often becomes non-Hausdorff even if the original étale groupoid is Hausdorff. Therefore, we treat not necessarily Hausdorff étale groupoids and their $\mathrm{C}^{*}$-algebras, which are defined by Connes [5]. As a byproduct, we obtain a simple proof that effectiveness of an étale groupoid is implied by a Cuntz-Krieger uniqueness theorem for a universal groupoid $C^{*}$-algebra (see Corollary 3.16). We remark that this result has been shown in [3] for Hausdorff étale groupoids in a different way and the proof in [3] seems to work for non-Hausdorff étale groupoids.

This paper is organized as follows. Having recalled definitions and basic facts about not necessarily Hausdorff étale groupoids and their $\mathrm{C}^{*}$-algebras, in Section 2 we introduce the notion of quotient étale groupoids and show some applications. Using quotients, we obtain a simple proof that a Cuntz-Krieger uniqueness theorem for a universal groupoid $\mathrm{C}^{*}$-algebra implies effectiveness of an étale groupoid.

In Section 3, for an étale groupoid $G$, we construct an étale abelian group bundle $G^{\text {ab }}$ through quotients. Finally, we show that the abelianization of a groupoid $\mathrm{C}^{*}$ algebra is isomorphic to the $C^{*}$-algebra associated to $G^{\mathrm{ab}}$. Since the abelianization of a $\mathrm{C}^{*}$-algebra is commutative, it is isomorphic to $C_{0}(X)$ for some locally compact Hausdorff space $X$ by the Gelfand-Naimark theorem. We show that the abelianization of a groupoid $\mathrm{C}^{*}$-algebra $C^{*}(G)$ is isomorphic to $C_{0}\left(\widehat{G^{\mathrm{ab}}}\right)$, where $\widehat{G^{\mathrm{ab}}}$ is introduced in this paper.

We obtain some results by using quotients of étale groupoids, which are not necessarily Hausdorff. We hope that this paper will stimulate the study of nonHausdorff étale groupoids.

\section{2. Étale groupoids and groupoid $\mathrm{C}^{*}$-algebras}

In this section we recall the notions of étale groupoids and groupoid $\mathrm{C}^{*}$-algebras. We refer to [9, 10, 12] for details.

2.1. Étale groupoids. A groupoid is a set $G$ together with a distinguished subset $G^{(0)} \subset G$, source and range maps $s, r: G \rightarrow G^{(0)}$ and a multiplication

$$
G^{(2)}:=\{(\alpha, \beta) \in G \times G \mid s(\alpha)=r(\beta)\} \ni(\alpha, \beta) \mapsto \alpha \beta \in G
$$

such that

(1) for all $x \in G^{(0)}, s(x)=x$ and $r(x)=x$ hold,

(2) for all $\alpha \in G, \alpha s(\alpha)=r(\alpha) \alpha=\alpha$ holds,

(3) for all $(\alpha, \beta) \in G^{(2)}, s(\alpha \beta)=s(\beta)$ and $r(\alpha \beta)=r(\alpha)$ hold,

(4) if $(\alpha, \beta),(\beta, \gamma) \in G^{(2)}$, we have $(\alpha \beta) \gamma=\alpha(\beta \gamma)$, and

(5) for every $\gamma \in G$, there exists $\gamma^{\prime} \in G$ which satisfies $\left(\gamma^{\prime}, \gamma\right),\left(\gamma, \gamma^{\prime}\right) \in G^{(2)}, s(\gamma)=$ $\gamma^{\prime} \gamma$ and $r(\gamma)=\gamma \gamma^{\prime}$.

Since the element $\gamma^{\prime}$ in (5) is uniquely determined by $\gamma, \gamma^{\prime}$ is called the inverse of $\gamma$ and denoted by $\gamma^{-1}$. We call $G^{(0)}$ the unit space of $G$. A subgroupoid of $G$ is a subset of $G$ which is closed under inversion and multiplication. For $U \subset G^{(0)}$, we define 
$G_{U}:=s^{-1}(U)$ and $G^{U}:=r^{-1}(U)$. We define also $G_{x}:=G_{\{x\}}$ and $G^{x}:=G^{\{x\}}$ for $x \in G^{(0)}$. The isotropy bundle of $G$ is denoted by $\operatorname{Iso}(G):=\{\gamma \in G \mid s(\gamma)=r(\gamma)\}$. If $G$ satisfies $G=\operatorname{Iso}(G), G$ is called a group bundle over $G^{(0)}$. A group bundle $G$ is said to be abelian if $G_{x}$ is an abelian group for all $x \in G^{(0)}$.

A topological groupoid is a groupoid equipped with a topology where the multiplication and the inverse are continuous. Note that the source map and range map of a topological groupoid are continuous.

\section{Definition 2.1. A topological groupoid $G$ is said to be étale if}

(1) the unit space $G^{(0)} \subset G$ is a locally compact Hausdorff space with respect to the relative topology of $G$,

(2) the source map $s: G \rightarrow G^{(0)}$ is a local homeomorphism (that is, for all $\alpha \in G$, there exists an open neighborhood $U \subset G$ of $\alpha$ such that $s(U) \subset G^{(0)}$ is open and $\left.s\right|_{U}$ is a homeomorphism onto $\left.s(U)\right)$.

An étale topological groupoid is called an étale groupoid for short. In this paper, we assume that the unit space of an étale groupoid is a locally compact Hausdorff space. We do not assume that an étale groupoid is a Hausdorff space as a topological space.

Note that a local homeomorphism is an open map. If $s$ is a local homeomorphism, then $r$ is also a local homeomorphism since $r(\gamma)=s\left(\gamma^{-1}\right)$ holds for all $\gamma \in G$. By Definition 2.1, the family of all locally compact Hausdorff open subsets of $G$ is an open basis for the topology of $G$. An étale groupoid $G$ is said to be effective if $G^{(0)}=\operatorname{Iso}(G)^{\circ}$, where $\operatorname{Iso}(G)^{\circ}$ denotes the interior of $\operatorname{Iso}(G)$.

In some papers, the condition that the source map $s: G \rightarrow G^{(0)}$ is a local homeomorphism in Definition 2.1 is replaced by the condition that the source map $s: G \rightarrow G$ is a local homeomorphism. As in Proposition 2.2, these definitions are equivalent.

Proposition 2.2 [6, Proposition 3.2]. Let $G$ be an étale groupoid. The unit space $G^{(0)}$ is an open subset of $G$. In particular, the source and range maps $s, r$ are local homeomorphisms as maps from $G$ to $G$.

Definition 2.3. Let $G$ be an étale groupoid. A subset $U \subset G$ is called a bisection if both $\left.s\right|_{U}$ and $\left.r\right|_{U}$ are injective.

For an étale groupoid $G$, an open bisection of $G$ is a locally compact Hausdorff space because it is homeomorphic to an open subset of $G^{(0)}$ and we assume that $G^{(0)}$ is locally compact Hausdorff. Note that the set of all open bisections forms a basis of $G$.

To a given discrete group action, we can associate an étale groupoid, which is called a transformation groupoid. We will consider a certain quotient of a transformation groupoid in Example 3.18.

Example 2.4. Let $X$ be a locally compact Hausdorff space, $\Gamma$ be a discrete group and $\alpha: \Gamma \curvearrowright X$ be an action. The transformation groupoid $\Gamma \ltimes_{\alpha} X$ is defined as follows. Define $\Gamma \ltimes_{\alpha} X:=\Gamma \times X$ as a topological space. The unit space of $\Gamma \ltimes_{\alpha} X$ is $X$, which 
is identified with the subset of $\Gamma \ltimes_{\alpha} X$ via an inclusion $X \ni x \mapsto(e, x) \in \Gamma \ltimes_{\alpha} X$. The source map and range map are defined by $s((t, x))=x$ and $r((t, x))=\alpha_{t}(x)$ respectively, for $(t, x) \in \Gamma \ltimes_{\alpha} X$. For a pair $\left(t_{1}, y\right),\left(t_{2}, x\right) \in \Gamma \ltimes_{\alpha} X$ with $y=\alpha_{t_{2}}(x)$, their multiplication is defined by $\left(t_{1}, y\right) \cdot\left(t_{2}, x\right):=\left(t_{1} t_{2}, x\right)$. An inverse is given by $(t, x)^{-1}=\left(t^{-1}, \alpha_{t}(x)\right)$. Then $\Gamma \ltimes_{\alpha} X$ is a Hausdorff étale groupoid.

Proposition 2.5 [9, Proposition 2.2.4]. Let $G$ be an étale groupoid and $U, V \subset G$ be open sets. Then a set $U V:=\{\alpha \beta \in G \mid \alpha \in U, \beta \in V, s(\alpha)=r(\beta)\} \subset G$ is an open set. Furthermore, if $U, V \subset G$ are open bisections, $U V$ is also an open bisection.

Definition 2.6. Let $G$ be a groupoid. A subset $F \subset G^{(0)}$ is said to be invariant if $s(\gamma) \in F$ implies $r(\gamma) \in F$ for all $\gamma$. A point $x \in G^{(0)}$ is called a fixed point if $\{x\} \subset G^{(0)}$ is invariant.

Note that a set $F \subset G^{(0)}$ is invariant if and only if $G^{(0)} \backslash F$ is invariant. If $F \subset G^{(0)}$ is invariant, then $G_{F}=G_{F} \cap G^{F} \subset G$ is a subgroupoid whose unit space is $F$.

Proposition 2.7. Let $G$ be an étale groupoid. Then the set of all fixed points $F \subset G^{(0)}$ is a closed subset.

Proof. We show that $G^{(0)} \backslash F \subset G^{(0)}$ is an open set. Take $x \in G^{(0)} \backslash F$. Then there exists $\gamma \in G$ such that $x=s(\gamma)$ and $x \neq r(\gamma)$. Take an open bisection $U$ which contains $\gamma$. Let $S_{U}: s(U) \rightarrow r(U)$ denote a homeomorphism defined by $S_{U}(s(\alpha))=r(\alpha)$ for each $\alpha \in U$. Since $G^{(0)}$ is Hausdorff, there exist open sets $U_{1}, V_{1} \subset G^{(0)}$ such that $s(\gamma) \in U_{1}$ , $r(\gamma) \in V_{1}$ and $U_{1} \cap V_{1}=\emptyset$. By the continuity of $S_{U}$, there exists an open set $U_{2} \subset U$ such that $\gamma \in U_{2}$ and $S_{U}\left(U_{2}\right) \subset V_{1}$. Now one can see that $U_{2} \subset G^{(0)} \backslash F$. Therefore, $G^{(0)} \backslash F \subset G^{(0)}$ is an open set.

We will use the next proposition for the set of all fixed points.

Proposition 2.8. Let $G$ be an étale groupoid and $U, F \subset G^{(0)}$ be an invariant open and closed subset, respectively. Then $G_{U} \subset G$ is an open subgroupoid of $G$ and an étale groupoid in the relative topology. Similarly, $G_{F} \subset G$ is a closed subgroupoid of $G$ and an étale groupoid in the relative topology.

Proof. Observe that $U$ and $F$ are locally compact Hausdorff spaces in the relative topology of $G^{(0)}$. Now it is clear that $G_{U}$ and $G_{F}$ are étale groupoids.

In particular, if $x \in G^{(0)}$ is a fixed point, then $G_{x} \subset G$ is a discrete group.

2.2. Étale groupoid $\mathbf{C} *$-algebras. Following Connes' idea in [5], we associate a $\mathrm{C}^{*}$-algebra to an étale groupoid which is not necessarily Hausdorff. See [6, Section 3] for more details.

Let $G$ be an étale groupoid. For an open Hausdorff subset $U \subset G$, we denote the set of all continuous functions with compact support on $U$ by $C_{c}(U)$. We regard an element in $C_{c}(U)$ as an element in $\operatorname{Funct}(G)$, the vector space of all complex-valued functions on $G$, by defining it to be 0 outside of $U$. We define $C(G):=\operatorname{span} \bigcup_{U} C_{c}(U) \subset$ Funct $(G)$, where the union is taken over all open Hausdorff subsets $U \subset G$. 
If $G$ is Hausdorff, then $C(G)$ coincides with $C_{c}(G)$. If $G$ is not Hausdorff, an element in $C(G)$ need not be continuous.

Proposition 2.9 [6, Proposition 3.10]. Let $G$ be an étale groupoid. Take an open basis $\left\{U_{i}\right\}_{i \in I}$ of $G$ consisting of open Hausdorff subsets. Then $C(G)$ is the linear span of $\bigcup_{i \in I} C_{c}\left(U_{i}\right)$. In particular, $C(G)$ is the linear span of $\cup_{U} C_{c}(U)$, where the union is taken over all open bisections of $G$.

Proof. This follows from a partition of unity argument.

Definition 2.10. Let $G$ be an étale groupoid. Recall that $C(G)$ is equipped with a structure of $\mathbb{C}$-vector space by pointwise addition and scalar multiplication. The multiplication $f * g \in C(G)$ and involution $f^{*} \in \mathcal{C}(G)$ of $f, g \in C(G)$ are defined by

$$
f * g(\gamma)=\sum_{\beta \in G_{s(\gamma)}} f\left(\gamma \beta^{-1}\right) g(\beta), \quad f^{*}(\gamma)=\overline{f\left(\gamma^{-1}\right)} .
$$

Then $C(G)$ is a *-algebra under these operations.

One can see that $C_{c}\left(G^{(0)}\right) \subset C(G)$ is a *-subalgebra.

Lemma 2.11 [6, Proposition 3.14]. Let $G$ be an étale groupoid and $f \in C(G)$. Then there exists $C_{f} \geq 0$ such that $\|\rho(f)\| \leq C_{f}$ for all Hilbert spaces $H$ and all *-homomorphisms $\rho: C(G) \rightarrow B(H)$.

Proof. We may assume that $f \in C_{c}(U)$ for some open bisection $U \subset G$. One can see that $f^{*} * f \in C_{c}\left(G^{(0)}\right)$. Since $C_{c}\left(G^{(0)}\right)$ is a union of commutative $\mathrm{C}^{*}$-algebras, we have $\|\rho(h)\| \leq \sup _{x \in G^{(0)}}|h(x)|$ for all $h \in C_{c}\left(G^{(0)}\right)$. Then we obtain $\|\rho(f)\|^{2}=\left\|\rho\left(f^{*} * f\right)\right\| \leq$ $\sup _{x \in G^{(0)}}\left|f^{*} * f(x)\right|<\infty$.

Let $G$ be an étale groupoid. We denote the left regular representation by $\lambda_{x}: C(G) \rightarrow$ $\ell^{2}\left(G_{x}\right)$ at $x \in G^{(0)}$, which is defined by

$$
\lambda_{x}(f) \delta_{\beta}=\sum_{s(\alpha)=r(\beta)} f(\alpha) \delta_{\alpha \beta}
$$

for $f \in C(G)$ and $\beta \in G_{x}$. One can see that $\bigoplus_{x \in G^{(0)}} \lambda_{x}$ is a faithful *-representation of $C(G)$. The reduced norm of $f \in C(G)$ is defined by

$$
\|f\|:=\sup _{x \in G^{(0)}}\left\|\lambda_{x}(f)\right\| .
$$

We denote the reduced groupoid $C^{*}$-algebra of $G$ by $C_{\lambda}^{*}(G)$, which is the completion of $C(G)$ by the reduced norm.

The universal norm of $f \in C(G)$ is defined by

$$
\|f\|:=\sup \{\|\rho(f)\| \mid \rho: C(G) \rightarrow B(H) \text { is a *-representation }\} .
$$

By Lemma 2.11, the universal norm takes values in $[0, \infty)$. Since the left regular representation of $C(G)$ induces a faithful *-representation of $C(G)$, the universal 
norm becomes a $C^{*}$-norm (see [4, Section 4]). The completion of $C(G)$ by universal norm is denoted by $C^{*}(G)$. We remark that every *-representation of $C(G)$ induces the *-representation of $C^{*}(G)$. Note that the inclusion $C_{c}\left(G^{(0)}\right) \subset C(G)$ extends to $C_{0}\left(G^{(0)}\right) \subset C^{*}(G)$.

Proposition 2.12. Let $G$ be an étale groupoid and $F \subset G^{(0)}$ be a closed invariant set. Then the restriction $\left.C(G) \ni f \mapsto f\right|_{G_{F}} \in C\left(G_{F}\right)$ extends to the surjective *-homomorphism $C^{*}(G) \rightarrow C^{*}\left(G_{F}\right)$.

Proof. First, we check that $\left.f\right|_{G_{F}} \in C\left(G_{F}\right)$ for all $f \in C(G)$. We may assume that $f \in C_{c}(U)$ for some open Hausdorff subset $U \subset G$, since $C(G)$ is spanned by $\cup_{U} C_{c}(U)$, where the union is taken over all open Hausdorff subsets $U \subset G$. Defining $V:=$ $G_{F} \cap U, V$ is a Hausdorff open subset of $G_{F}$. Then $\left.f\right|_{G_{F}}$ is contained in $C_{c}(V) \subset C\left(G_{F}\right)$.

Direct calculations imply that the restriction $\left.C(G) \ni f \mapsto f\right|_{G_{F}} \in C\left(G_{F}\right)$ is a *-homomorphism.

Next, we show that the restriction $\left.C(G) \ni f \mapsto f\right|_{G_{F}} \in C\left(G_{F}\right)$ is surjective. Note that $\left\{G_{F} \cap U \mid U \subset G\right.$ is an open Hausdorff subset $\}$ is an open basis of $G_{F}$. Take an open Hausdorff subset $U \subset G_{F}$ and $f \in C_{c}\left(G_{F} \cap U\right)$ arbitrarily. Put $V:=G_{F} \cap U$. Since $V \subset U$ is a closed subset of $U$ and $f \in C_{c}(V)$, there exists $\tilde{f} \in C_{c}(U)$ such that $\left.\tilde{f}\right|_{V}=f$ by the Tietze extension theorem. Now we obtain $\tilde{f} \in C(G)$ such that $\left.\tilde{f}\right|_{G_{F}}=f$. By Proposition 2.9, $C\left(G_{F}\right)$ is the linear span of $\bigcup_{U} C_{c}\left(G_{F} \cap U\right)$, where the union is taken over all open Hausdorff subsets $U \subset G$. Therefore, the restriction $\left.C(G) \ni f \mapsto f\right|_{G_{F}} \in C\left(G_{F}\right)$ is surjective.

By the universality of $C^{*}(G)$, the restriction $\left.C(G) \ni f \mapsto f\right|_{G_{F}} \in C\left(G_{F}\right)$ extends to the *-homomorphism $C^{*}(G) \rightarrow C^{*}\left(G_{F}\right)$. Since the image of $C^{*}(G)$ is dense in $C^{*}\left(G_{F}\right)$, $C^{*}(G) \rightarrow C^{*}\left(G_{F}\right)$ is surjective.

\section{Quotients of étale groupoids}

After introducing the notion of quotient étale groupoids, we will see that a quotient of an étale groupoid induces a *-homomorphism of a groupoid $\mathrm{C}^{*}$-algebra.

3.1. Quotients of étale groupoids. In this subsection we introduce the notion of quotient étale groupoids. First, we define normal subgroupoids and quotient groupoids. Then we show that quotient groupoids of étale groupoids by open normal subgroupoids again become étale.

Definition 3.1. Let $G$ be a groupoid. A subgroupoid $H \subset G$ is said to be normal if

(1) $G^{(0)} \subset H \subset \operatorname{Iso}(G)$ holds and

(2) $\alpha H \alpha^{-1} \subset H$ holds for all $\alpha \in G$.

Definition 3.2. Let $G$ be a groupoid and $H \subset G$ be a normal subgroupoid. Then we define an equivalence relation $\sim$ on $G$ by declaring that $\alpha \sim \beta$ if $s(\alpha)=s(\beta)$ and $\alpha \beta^{-1} \in H$. We denote the quotient set $G / \sim$ by $G / H$. 
We prove some lemmas needed to define the groupoid structure of a quotient groupoid.

Lemma 3.3. Let $G$ be a groupoid and $H \subset G$ be a normal subgroupoid. Suppose that $\alpha, \alpha^{\prime} \in G$ satisfy $\alpha \sim \alpha^{\prime}$. Then we have $s(\alpha)=s\left(\alpha^{\prime}\right)$ and $r(\alpha)=r\left(\alpha^{\prime}\right)$.

Proof. It follows that $s(\alpha)=s\left(\alpha^{\prime}\right)$ from the definition of $\alpha \sim \alpha^{\prime}$. Since $\alpha \alpha^{\prime-1} \in H \subset$ Iso $(G)$, we have $r(\alpha)=r\left(\alpha \alpha^{\prime-1}\right)=s\left(\alpha \alpha^{\prime-1}\right)=r\left(\alpha^{\prime}\right)$.

Lemma 3.4. Let $G$ be a groupoid and $H \subset G$ be a normal subgroupoid. Suppose that $\alpha, \alpha^{\prime}, \beta, \beta^{\prime} \in G$ satisfy $\alpha \sim \alpha^{\prime}, \beta \sim \beta^{\prime}, s(\alpha)=r(\beta)$. Then we have $s\left(\alpha^{\prime}\right)=r\left(\beta^{\prime}\right)$ and $\alpha \beta \sim \alpha^{\prime} \beta^{\prime}$.

Proof. By Lemma 3.3, we have $s(\alpha)=s\left(\alpha^{\prime}\right)$ and $r(\beta)=r\left(\beta^{\prime}\right)$. Using $s(\alpha)=r(\beta)$, we obtain $s\left(\alpha^{\prime}\right)=r\left(\beta^{\prime}\right)$.

The last assertion follows from a simple calculation. Indeed, we have $s(\alpha \beta)=s(\beta)=$ $s\left(\beta^{\prime}\right)=s\left(\alpha^{\prime} \beta^{\prime}\right)$ and

$$
\alpha \beta\left(\alpha^{\prime} \beta^{\prime}\right)^{-1}=\alpha \beta \beta^{-1} \alpha^{\prime-1}=\left(\alpha \beta \beta^{-1} \alpha^{-1}\right)\left(\alpha \alpha^{\prime-1}\right) \in H .
$$

Note that $\alpha \beta \beta^{\prime-1} \alpha^{-1} \in H$, since $H$ is normal.

Definition 3.5. Let $G$ be a groupoid, $H \subset G$ be a normal subgroupoid and $q$ : $G \rightarrow G / H$ be the quotient map. A groupoid structure of $G / H$ is defined as follows:

- $\quad$ a unit space $(G / H)^{(0)}$ is $q\left(G^{(0)}\right)$, which can be identified with $G^{(0)}$ via an injection $\left.q\right|_{G^{(0)}}$;

- $\quad$ source and range maps $s, r: G / H \rightarrow G^{(0)}$ are defined by $s(q(\gamma)):=q(s(\gamma))$, $r(q(\gamma)):=q(r(\gamma))$ for $\gamma \in G$

- multiplication of $G / H$ is defined by $q(\alpha) q(\beta):=q(\alpha \beta)$ for $\alpha, \beta \in G$ with $s(\alpha)=$ $r(\beta)$.

One can see that the inverse map of $G / H$ satisfies $q(\gamma)^{-1}=q\left(\gamma^{-1}\right)$ for $\gamma \in G$. Then $G / H$ is a groupoid under these operations.

Remark 3.6. The operations of $G / H$ are well defined by Lemmas 3.3 and 3.4.

If $G$ is a topological groupoid, then we consider the quotient topology as a topology of $G / H$.

Lemma 3.7. Let $G$ be an étale groupoid and $H \subset G$ be an open normal subgroupoid. Then the quotient map $q: G \rightarrow G / H$ is an open map. Furthermore, $q$ is a local homeomorphism.

Proof. Let $U \subset G$ be an open subset. Then $q^{-1}(q(U))=U H$ is an open subset of $G$ by Proposition 2.5. Hence, $q(U) \subset G / H$ is an open subset by the definition of the quotient topology.

Next, we show that the quotient map $q: G \rightarrow G / H$ is a local homeomorphism. Fix a $\gamma \in G$. Then take an open bisection $U \subset G$ with $\gamma \in U$. One can see that $\left.q\right|_{U}$ is injective. Since $q$ is an open map, $\left.q\right|_{U}$ is a homeomorphism onto an open subset $q(U) \subset G$. Hence, $q$ is a local homeomorphism. 
Observe that $\left.q\right|_{G^{(0)}}: G^{(0)} \rightarrow(G / H)^{(0)}$ is homeomorphic.

Proposition 3.8. Let $G$ be an étale groupoid and $H \subset G$ be an open normal subgroupoid. Then $G / H$ is an étale groupoid.

Proof. First, we show the continuity of the inverse $G / H \ni \delta \mapsto \delta^{-1} \in G / H$. One can see that a map $G \ni \gamma \mapsto q(\gamma)^{-1} \in G / H$ is continuous, since the following diagram is commutative:

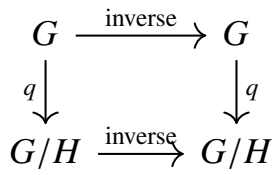

By the definition of the quotient topology, the inverse of $G / H$ is continuous.

Next, we show that the multiplication of $G / H$ is continuous. Take $(q(\alpha), q(\beta)) \in$ $(G / H)^{(2)}$ and an open set $U \subset G / H$ such that $q(\alpha) q(\beta) \in U$. Since $\alpha \beta \in q^{-1}(U)$ and $q^{-1}(U) \subset G$ is open, there exist open sets $V_{1}, V_{2} \subset G$ such that $\alpha \in V_{1}, \beta \in V_{2}$ and $V_{1} V_{2} \subset q^{-1}(U)$. Subsets $V_{1}, V_{2} \subset G$ are open, so $q\left(V_{1}\right), q\left(V_{2}\right) \subset G / H$ are open. One can see that $q(\alpha) \in q\left(V_{1}\right), q(\beta) \in q\left(V_{2}\right)$ and $q\left(V_{1}\right) q\left(V_{2}\right)=q\left(V_{1} V_{2}\right) \subset U$. Therefore, the multiplication of $G / H$ is continuous.

Finally, we show that $G / H$ is étale. Since the restriction $\left.q\right|_{G^{(0)}}$ gives a homeomorphism from $G^{(0)}$ to $(G / H)^{(0)},(G / H)^{(0)}$ is a locally compact Hausdorff space. One can see that the source map $s: G / H \rightarrow(G / H)^{(0)}$ is a local homeomorphism, since we have Lemma 3.7 and the following diagram is commutative for every open bisection $U \subset G$ :

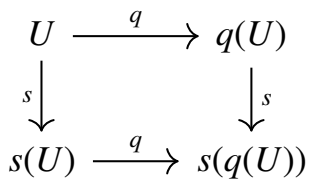

Therefore, $G / H$ is an étale groupoid.

Definition 3.9. Let $G_{1}$ and $G_{2}$ be groupoids. A map $\Phi: G_{1} \rightarrow G_{2}$ is called a groupoid homomorphism if $(\Phi(\alpha), \Phi(\beta)) \in G_{2}^{(2)}$ and $\Phi(\alpha \beta)=\Phi(\alpha) \Phi(\beta)$ hold for all $(\alpha, \beta) \in G_{1}^{(2)}$.

We obtain the next theorem by Lemma 3.7 and Proposition 3.8.

THeOREM 3.10. Let $G$ be an étale groupoid and $H \subset G$ be an open normal subgroupoid. Then the sequence of the étale groupoids

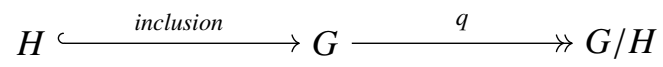

is exact, that is, $q^{-1}\left((G / H)^{(0)}\right)=H$.

As in the case of topological groups, Hausdorffness of a quotient groupoid can be characterized as follows. 
Proposition 3.11. Let $G$ be an étale groupoid and $H \subset G$ be an open normal subgroupoid. Then $G / H$ is Hausdorff if and only if $H \subset G$ is closed.

Proof. Recall that an étale groupoid $G$ is Hausdorff if and only if its unit space $G^{(0)}$ is a closed subset of $G$ (see, for example, [12, Lemma 2.3.2]). If $G / H$ is Hausdorff, $(G / H)^{(0)} \subset G / H$ is closed. Hence, $H=q^{-1}\left((G / H)^{(0)}\right)$ is a closed subset of $G$.

Suppose that $H \subset G$ is closed. Since $q$ is an open map, $(G / H) \backslash(G / H)^{(0)}=q(G \backslash$ $H) \subset G / H$ is open. Hence, $(G / H)^{(0)} \subset G / H$ is closed, which implies that $G / H$ is Hausdorff.

Proposition 3.12. Let $G$ be an étale groupoid. Then the interior of isotropy $\operatorname{Iso}(G)^{\circ} \subset$ $\operatorname{Iso}(G)$ is a normal subgroupoid.

Proof. We show that $\operatorname{Iso}(G)^{\circ}$ is normal. By Proposition 2.2, $G^{(0)}$ is contained in $\operatorname{Iso}(G)^{\circ}$. Take $\alpha \in G$ and $\gamma \in \operatorname{Iso}(G)^{\circ}$ with $s(\alpha)=r(\gamma)$. There exist open bisections $U, V \subset G$ with $\alpha \in U$ and $\gamma \in V \subset \operatorname{Iso}(G)$. Then, by Proposition 2.5, $U V U^{-1} \subset G$ is an open subset which contains $\alpha \gamma \alpha^{-1}$. Since $U$ is bisection and $V \subset \operatorname{Iso}(G)$, we have $U V U^{-1} \subset \operatorname{Iso}(G)$. Therefore, $\alpha \gamma \alpha^{-1} \in \operatorname{Iso}(G)^{\circ}$ and $\operatorname{Iso}(G)^{\circ}$ is an open normal subgroupoid.

An étale groupoid $G / \operatorname{Iso}(G)^{\circ}$, which is a special case of quotient groupoids, coincides with a groupoid of germs of the canonical action (see [11, Section 3]). One can see that $G / \operatorname{Iso}(G)^{\circ}$ is effective.

3.2. *-homomorphisms induced by quotients of étale groupoids. For an étale groupoid $G$ and an open normal subgroupoid $H \subset G$, we have obtained the quotient étale groupoid $G / H$. Next, we see that the quotient map $q: G \rightarrow G / H$ induces a *-homomorphism $C^{*}(G) \rightarrow C^{*}(G / H)$.

For $f \in C(G)$, we define $\tilde{f}: G / H \rightarrow \mathbb{C}$ by

$$
\tilde{f}(\gamma):=\sum_{q(\alpha)=\gamma} f(\alpha)
$$

for $\gamma \in G / H$. Then the following proposition holds.

Proposition 3.13. Let $G$ be an étale groupoid and $H \subset G$ be an open normal subgroupoid. Then $C(G) \ni f \mapsto \tilde{f} \in C(G / H)$ is a surjective *-homomorphism.

Proof. First, we show $\tilde{f} \in C(G / H)$. We may assume that there exists an open bisection $U \subset G$ such that $\left.f\right|_{U} \in C_{c}(U)$ and $\left.f\right|_{G \backslash U}=0$. Then $q(U) \subset G / H$ is an open bisection and $\left.\tilde{f}\right|_{q(U)}=f \circ\left(\left.q\right|_{U}\right)^{-1} \in C_{c}(q(U))$, since $\left.q\right|_{U}$ is a homeomorphism onto the image. Moreover, one can see that $\tilde{f}_{(G / H) \backslash q(U)}=0$. Hence, $\tilde{f} \in C_{c}(q(U)) \subset C(G / H)$.

We show that $C(G) \ni f \mapsto \tilde{f} \in \mathcal{C}(G / H)$ is a *homomorphism. We only check that $C(G) \ni f \mapsto \tilde{f} \in C(G / H)$ preserves multiplication, since it is easy to check that this 
map is linear and preserves the involution. For all $f, g \in C(G)$ and $\gamma^{\prime} \in G / H$, we have

$$
\begin{aligned}
\widetilde{f * g}\left(\gamma^{\prime}\right) & =\sum_{q(\gamma)=\gamma^{\prime}} f * g(\gamma)=\sum_{q(\gamma)=\gamma^{\prime}} \sum_{\alpha \beta=\gamma} f(\alpha) g(\beta)=\sum_{q(\alpha \beta)=\gamma^{\prime}} f(\alpha) g(\beta), \\
\tilde{f} * \tilde{g}\left(\gamma^{\prime}\right) & =\sum_{\alpha^{\prime} \beta^{\prime}=\gamma^{\prime}} \tilde{f}\left(\alpha^{\prime}\right) \tilde{g}\left(\beta^{\prime}\right)=\sum_{\alpha^{\prime} \beta^{\prime}=\gamma^{\prime}} \sum_{q(\alpha)=\alpha^{\prime}} \sum_{q(\beta)=\beta^{\prime}} f(\alpha) g(\beta) \\
& =\sum_{q(\alpha \beta)=\gamma^{\prime}} f(\alpha) g(\beta) .
\end{aligned}
$$

Finally, we show that $C(G) \ni f \mapsto \tilde{f} \in \mathcal{C}(G / H)$ is surjective. Note that

$$
\{q(U) \subset G / H \mid U \subset G \text { is an open bisection }\}
$$

is an open basis of $G$. Let $U \subset G$ be an open bisection and $f \in C_{c}(q(U))$. One can see that $\left.q\right|_{U}$ is a homeomorphism onto its image. Define $g:=\left.f \circ q\right|_{U} \in C_{c}(U)$. Then we have $\widetilde{g}=f$. By Proposition 2.12, $C(G / H)$ is the linear span of $\bigcup_{U} C_{c}(q(U))$, where the union is taken over all open bisections $U \subset G$. Hence, $C(G) \ni f \mapsto \tilde{f} \in C(G / H)$ is a surjective *-homomorphism.

By Proposition 3.13, a map $C(G) \ni f \mapsto \tilde{f} \in C(G / H) \subset C^{*}(G / H)$ is a *homomorphism. By the definition of the universal norm of $C(G)$, we have $\|\tilde{f}\| \leq\|f\|$ for all $f \in C(G)$. Therefore the *-homomorphism in Proposition 3.13 extends to the *-homomorphism $Q: C^{*}(G) \rightarrow C^{*}(G / H)$. Since the image of $Q$ is dense in $C^{*}(G / H)$, $Q$ is surjective (see, for example, [2, Corollary II.5.1.2]).

We make some observations on a Cuntz-Krieger uniqueness theorem in the remainder of this section.

Lemma 3.14. Let $Q: C^{*}(G) \rightarrow C^{*}(G / H)$ be the *-homomorphism as above. Then $\operatorname{ker} Q \cap C_{0}\left(G^{(0)}\right)=\{0\}$ holds.

Proof. Since the universal norm of a function in $C_{c}\left(G^{(0)}\right)$ coincides with the supremum norm, $\left.Q\right|_{C_{c}\left(G^{(0)}\right)}$ is isometric. Therefore, $\left.Q\right|_{C_{0}\left(G^{(0)}\right)}$ is isometric and $\operatorname{ker} Q \cap C_{0}\left(G^{(0)}\right)=$ $\{0\}$.

Lemma 3.15. Let $G$ be an étale groupoid and $H \subset G$ be an open normal subgroupoid. Then the *-homomorphism $Q: C^{*}(G) \rightarrow C^{*}(G / H)$ induced by Proposition 3.13 is injective if and only if $H=G^{(0)}$.

Proof. It is clear that the *-homomorphism $Q: C^{*}(G) \rightarrow C^{*}(G / H)$ is injective if $H=G^{(0)}$. Suppose that $G^{(0)} \subsetneq H$ and take $\gamma_{0} \in H \backslash G^{(0)}$. There exists an open bisection $U \subset G$ with $\gamma_{0} \in U \subset H$. By the Urysohn lemma, there exists $f_{1} \in C_{c}(U)$ with $f_{1}\left(\gamma_{0}\right)=$ 1. Define $f_{2} \in C_{c}\left(G^{(0)}\right)$ by

$$
f_{2}(\gamma)= \begin{cases}f_{1} \circ\left(\left.s\right|_{U}\right)^{-1}(\gamma) & (\gamma \in s(U)), \\ 0 & \left(\gamma \in G^{(0)} \backslash s(U)\right) .\end{cases}
$$

We have $f:=f_{1}-f_{2} \neq 0$, since $f\left(\gamma_{0}\right)=1$. One can see that $Q(f)=0$, which means that $Q$ is not injective. 


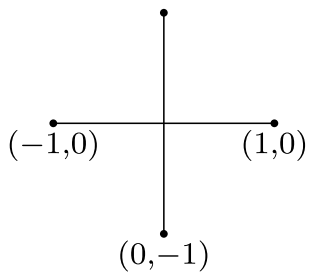

Figure 1. Picture of $X$ in Example 3.18.

Recall that an étale groupoid $G$ is said to be effective if $G^{(0)}=\operatorname{Iso}(G)^{\circ}$.

Corollary 3.16 (cf. [3, Proposition 5.5]). Let $G$ be an étale groupoid. Assume that every nonzero ideal $I \subset C^{*}(G)$ satisfies $I \cap C_{0}\left(G^{(0)}\right) \neq\{0\}$. Then $G$ is effective.

Proof. By Proposition 3.12, $\operatorname{Iso}(G)^{\circ}$ is a normal subgroupoid of $G$. Letting $Q: C^{*}(G) \rightarrow C^{*}\left(G / \operatorname{Iso}(G)^{\circ}\right)$ be the *-homomorphism induced by Proposition 3.13, we have $\operatorname{ker} Q \cap C_{0}\left(G^{(0)}\right)=\{0\}$ by Lemma 3.14. The assumption implies that $Q: C^{*}(G) \rightarrow$ $C^{*}\left(G / \operatorname{Iso}(G)^{\circ}\right)$ is injective. Therefore, we obtain $\operatorname{Iso}(G)^{\circ}=G^{(0)}$ by Lemma 3.15.

Remark 3.17. It was proved in [3, Proposition 5.5] that Corollary 3.16 holds for Hausdorff étale groupoids. In the proposition, the authors use the augmentation representation, which seems to work for non-Hausdorff étale groupoids.

As shown in Proposition 3.13, the quotient map $G \rightarrow G / \operatorname{Iso}(G)^{\circ}$ of étale groupoids induces the *-homomorphism $C^{*}(G) \rightarrow C^{*}\left(G / \operatorname{Iso}(G)^{\circ}\right)$. Using this *-homomorphism, we obtain the proof of Corollary 3.16, which seems to be more direct than that in $[3$, Proposition 5.5].

The converse of Corollary 3.16 does not hold for non-Hausdorff étale groupoids. Indeed, Exel showed that there exists an effective étale groupoid $G$ such that there exists a nonzero ideal $I \subset C^{*}(G)$ with $I \cap C_{0}\left(G^{(0)}\right) \neq\{0\}$ in [7] (cf. Example 3.18).

Example $3.18\left[7\right.$, Section 2]. Let $X:=([-1,1] \times\{0\}) \cup(\{0\} \times[-1,1]) \subset \mathbb{R}^{2}$ (see Figure 1$)$ and $K:=\{e, s, t, s t\}$ be the Klein group, which is isomorphic to $\mathbb{Z} / 2 \mathbb{Z} \oplus \mathbb{Z} / 2 \mathbb{Z}$. We define an action $\sigma$ of $K$ on $X$ by

$$
\sigma_{s}((x, y))=(-x, y), \quad \sigma_{t}((x, y))=(x,-y), \quad \sigma_{s t}((x, y))=(-x,-y)
$$

for $(x, y) \in X$.

Consider the transformation groupoid $G:=K \ltimes_{\sigma} X$ (see Example 2.4). One can see that

$$
\begin{aligned}
\operatorname{Iso}(G)=G^{(0)} & \cup\{(s,(0, y)) \in G \mid y \in[-1,1]\} \\
& \cup\{(t,(x, 0)) \in G \mid x \in[-1,1]\} \cup\{(\text { st },(0,0))\} .
\end{aligned}
$$

Moreover, we have $\operatorname{Iso}(G)^{\circ}=\operatorname{Iso}(G) \backslash\{(s,(0,0)),(t,(0,0)),(s t,(0,0))\}$. Since $\operatorname{Iso}(G)^{\circ}$ is not closed in $G$ (for example, $\left.(s,(0,0)) \in \overline{\operatorname{Iso}(G)^{\circ}} \backslash \operatorname{Iso}(G)^{\circ}\right)$, the quotient étale 
groupoid $G / \operatorname{Iso}(G)^{\circ}$ is not Hausdorff by Proposition 3.11. In [7], Exel shows that there exists a nonzero ideal $I \subset C^{*}\left(G / \operatorname{Iso}(G)^{\circ}\right)$ with $I \cap C_{0}\left(\left(G / \operatorname{Iso}(G)^{\circ}\right)^{(0)}\right) \neq\{0\}$, although it is effective.

Let $G$ be an étale groupoid. In [4], the authors defined the notion of singularity for an element of $C_{\lambda}^{*}(G)$. An element $a \in C_{\lambda}^{*}(G)$ is said to be singular if the interior of $\left\{\gamma \in G \mid\left\langle\delta_{\gamma} \mid \lambda_{s(\gamma)}(a) \delta_{s(\gamma)}\right\rangle \neq 0\right\}$ is empty, where $\delta_{\gamma} \in \ell^{2}\left(G_{x}\right)$ denotes the delta function at $\gamma \in G_{x}$. In [4], the authors proved the following theorem.

Theorem 3.19 [4, Theorem 4.4]. Let G be a second countable étale groupoid. Assume that $G$ is effective and $C_{\lambda}^{*}(G)$ has no nonzero singular element. Then every nonzero ideal $I \subset C_{\lambda}^{*}(G)$ satisfies $I \cap C_{0}\left(G^{(0)}\right) \neq\{0\}$.

By the universality of $C^{*}(G)$, the left representation extends to the *-representation $\lambda_{x}: C^{*}(G) \rightarrow B\left(\ell^{2}\left(G_{x}\right)\right)$. Following [4], we say that an element $a \in C^{*}(G)$ is singular if the interior of $\left\{\gamma \in G \mid\left\langle\delta_{\gamma} \mid \lambda_{s(\gamma)}(a) \delta_{s(\gamma)}\right\rangle \neq 0\right\}$ is empty, where $\delta_{\gamma} \in \ell^{2}\left(G_{x}\right)$ denotes the delta function at $\gamma \in G_{x}$. A uniqueness theorem for $C^{*}(G)$ implies that $C^{*}(G)$ has no nonzero singular elements.

Proposition 3.20. Let $G$ be a second countable étale groupoid. Assume that every nonzero ideal $I \subset C^{*}(G)$ satisfies $I \cap C_{0}\left(G^{(0)}\right) \neq\{0\}$. Then $C^{*}(G)$ has no nonzero singular elements.

Proof. Observe that the canonical surjective *-homomorphism $C^{*}(G) \rightarrow C_{\lambda}^{*}(G)$ is isomorphic by the assumption. Note that $G$ is effective by Proposition 3.16. We define $S:=\left\{x \in G^{(0)} \mid G_{x} \cap G^{x}=\{x\}\right\}$. One can see that $S$ is an invariant set. Moreover, $S$ is a dense subset of $G^{(0)}$ by [11, Proposition 3.6]. Therefore, letting $\pi:=\bigoplus_{x \in S} \lambda_{x}, \pi$ is injective on $C_{0}\left(G^{(0)}\right)$. Then $\pi$ is injective by the assumption.

Let $a \in C^{*}(G)$ be a singular element. By [4, Lemma 4.2], we have

$$
s\left(\left\{\gamma \in G \mid\left\langle\delta_{\gamma} \mid \lambda_{s(\gamma)}(a) \delta_{s(\gamma)}\right\rangle \neq 0\right\}\right) \subset G^{(0)} \backslash S .
$$

Using this fact, we show that $\pi(a)=0$. Take $x \in S$. Assume that there exist $\alpha, \beta \in G_{x}$ such that

$$
\left\langle\delta_{\alpha} \mid \lambda_{x}(a) \delta_{\beta}\right\rangle \neq 0
$$

Then we have

$$
\left\langle\delta_{\alpha \beta^{-1}} \mid \lambda_{s\left(\beta^{-1}\right)} \delta_{s\left(\beta^{-1}\right)}\right\rangle=\left\langle\delta_{\alpha} \mid \lambda_{x}(a) \delta_{\beta}\right\rangle \neq 0 .
$$

It follows that $r(\beta)=s\left(\beta^{-1}\right) \notin S$. This contradicts the fact that $x=s(\beta) \in S$ and $S$ is invariant. Now we have $\left\langle\delta_{\alpha} \mid \lambda_{x}(a) \delta_{\beta}\right\rangle=0$ for all $\alpha, \beta \in G_{x}$ and therefore $\lambda_{x}(a)=0$ holds for all $x \in S$. Now we have $\pi(a)=0$, which implies $a=0$. Hence, $C^{*}(G)$ has no nonzero singular element. 


\section{The abelianizations of étale groupoid $C *$-algebras}

In this section we calculate the abelianizations of étale groupoid $\mathrm{C}^{*}$-algebras. First, we recall the abelianizations of $\mathrm{C}^{*}$-algebras, following [1, Definition 2.8]. For a $C^{*}$-algebra $A$, its abelianization is defined by $A^{\mathrm{ab}}=A / I$, where $I \subset A$ is the closed two-sided ideal generated by $\{x y-y x \in A \mid x, y \in A\}$. The abelianization $A^{\text {ab }}$ is a commutative $\mathrm{C}^{*}$-algebra with the following universality: for all commutative $\mathrm{C}^{*}$ algebras $B$ and $*$-homomorphisms $\pi: A \rightarrow B$, there exists the unique $*$-homomorphism $\tilde{\pi}: A^{\mathrm{ab}} \rightarrow B$ such that $\tilde{\pi} \circ q=\pi$, where $q: A \rightarrow A^{\mathrm{ab}}$ denotes the quotient map.

4.1. One-dimensional representations of a groupoid $\mathbf{C}^{*}$-algebra. For a $\mathrm{C}^{*}$ algebra $A$, we denote the set of all one-dimensional nondegenerate representations of $A$ by $\Delta(A)$. Namely, $\Delta(A)$ is the set of all nonzero *-homomorphisms from $A$ to C. We suppose that $\Delta(A)$ is equipped with the pointwise convergence topology. If $A$ is commutative, $\Delta(A)$ is known as the Gelfand spectrum of $A$. First, we calculate $\Delta\left(C^{*}(G)\right)$.

Let $G$ be an étale groupoid and $x \in G^{(0)}$ be a fixed point of $G$. Note that $G_{x}$ is a discrete group. We temporarily denote the surjection in Proposition 2.12 by $Q_{x}: C^{*}(G) \rightarrow C^{*}\left(G_{x}\right)$. Also, we denote the circle group by $\mathbb{T}:=\{z \in \mathbb{C}|| z \mid=1\}$. For a group homomorphism $\chi: G_{x} \rightarrow \mathbb{T}$, a map $C_{c}\left(G_{x}\right) \ni f \mapsto \sum_{\gamma \in G_{x}} \chi(\gamma) f(\gamma) \in \mathbb{C}$ is a *-homomorphism. This *-homomorphism extends to the *-homomorphism $C^{*}\left(G_{x}\right) \rightarrow \mathbb{C}$, which we also denote by $\chi: C^{*}\left(G_{x}\right) \rightarrow \mathbb{C}$.

Definition 4.1. Let $G$ be an étale groupoid, $x \in G^{(0)}$ be a fixed point and $\chi: G_{x} \rightarrow \mathbb{T}$ be a group homomorphism. Then we define a $*$-homomorphism $\varphi_{x, \chi}: C^{*}(G) \rightarrow \mathbb{C}$ by $\varphi_{x, \chi}:=\chi \circ Q_{x}$.

We will show that all elements of $\Delta\left(C^{*}(G)\right)$ have this form (Theorem 4.8).

Proposition 4.2. Let $G$ be an étale groupoid and $\varphi \in \Delta\left(C^{*}(G)\right)$. Then there exists a unique $x_{\varphi} \in G^{(0)}$ that satisfies $\varphi(f)=f\left(x_{\varphi}\right)$ for all $f \in C_{0}\left(G^{(0)}\right)$.

Proof. We have $\left.\varphi\right|_{C_{0}\left(G^{0}\right)} \neq 0$ since $C_{0}\left(G^{(0)}\right)$ has an approximate identity of $C^{*}(G)$. Therefore, $\left.\varphi\right|_{C_{0}\left(G^{(0)}\right)}$ belongs to $\Delta\left(C_{0}\left(G^{(0)}\right)\right)$. Now the existence and uniqueness of $x_{\varphi} \in G^{(0)}$ follow from the Gelfand-Naimark theorem.

Proposition 4.3. Let $G$ be an étale groupoid and $\varphi \in \Delta\left(C^{*}(G)\right)$. Then $x_{\varphi} \in G^{(0)}$ as defined in Proposition 4.2 is a fixed point.

Proof. Assume that $\gamma \in G$ satisfies $s(\gamma)=x_{\varphi}$. We show that $r(\gamma)=x_{\varphi}$. There exists an open bisection $U \subset G$ with $\gamma \in U$. Take $n_{\gamma} \in C_{c}(U)$ which satisfies $n_{\gamma}(\gamma)=1$. Note that we have $n_{\gamma}^{*} * n_{\gamma} \in C_{c}\left(G^{(0)}\right)$ and $n_{\gamma}^{*} * n_{\gamma}\left(x_{\varphi}\right)=\left|n_{\gamma}(\gamma)\right|^{2}=1$. Fix $f \in C_{c}\left(G^{(0)}\right)$ arbitrarily.

Direct calculations show that $n_{\gamma}^{*} * f * n_{\gamma}\left(x_{\varphi}\right)=\overline{n_{\gamma}(\gamma)} f(r(\gamma)) n_{\gamma}(\gamma)=f(r(\gamma))$. On the other hand, one can see that $n_{\gamma}^{*} * f * n_{\gamma} \in C_{c}\left(G^{(0)}\right)$. Then we have

$$
n_{\gamma}^{*} * f * n_{\gamma}\left(x_{\varphi}\right)=\varphi\left(n_{\gamma}^{*} * f * n_{\gamma}\right)=\varphi\left(n_{\gamma}^{*}\right) \varphi(f) \varphi\left(n_{\gamma}\right)=\varphi\left(n_{\gamma}^{*} * n_{\gamma}\right) \varphi(f)=f\left(x_{\varphi}\right) .
$$

Therefore, $f(r(\gamma))=f\left(x_{\varphi}\right)$ holds for all $f \in C_{c}\left(G^{(0)}\right)$, which implies $r(\gamma)=x_{\varphi}$. Hence, $x_{\varphi} \in G^{(0)}$ is a fixed point of $G$. 
Proposition 4.4. Let $G$ be an étale groupoid, $\varphi \in \Delta\left(C^{*}(G)\right)$ and $\gamma \in G_{x_{\varphi}}$. Take an open bisection $U_{\gamma} \subset G$ with $\gamma \in U_{\gamma}$ and $f_{\gamma} \in C_{c}\left(U_{\gamma}\right)$ with $f_{\gamma}(\gamma)=1$. Then $\varphi\left(f_{\gamma}\right)$ is independent of the choice of $U_{\gamma}$ and $f_{\gamma}$. Moreover, we have $\varphi\left(f_{\gamma}\right) \in \mathbb{T}$.

Proof. First, we show that $\varphi\left(f_{\gamma}\right) \in \mathbb{T}$. Since $f_{\gamma}^{*} * f_{\gamma} \in C_{0}\left(G^{(0)}\right)$, we have

$$
\left|\varphi\left(f_{\gamma}\right)\right|^{2}=\varphi\left(f_{\gamma}^{*} * f_{\gamma}\right)=f_{\gamma}^{*} * f_{\gamma}\left(x_{\varphi}\right)=\left|f_{\gamma}(\gamma)\right|^{2}=1 .
$$

Therefore, $\varphi\left(f_{\gamma}\right) \in \mathbb{T}$.

Second, we show that $\varphi\left(f_{\gamma}\right)$ is independent of the choice of $U_{\gamma}$ and $f_{\gamma}$. Assume that $f_{\gamma} \in C_{c}\left(U_{\gamma}\right)$ and $g_{\gamma} \in C_{c}\left(V_{\gamma}\right)$ satisfy $f_{\gamma}(\gamma)=g_{\gamma}(\gamma)=1$, where $U_{\gamma}$ and $V_{\gamma} \subset G$ are open bisections. Find a function $h \in C_{c}\left(s\left(U_{\gamma} \cap V_{\gamma}\right)\right) \subset C_{c}\left(G^{(0)}\right)$ such that $h(s(\gamma))=1$. Recall that $s(\gamma)=r(\gamma)=x_{\varphi}$ since $x_{\varphi}$ is a fixed point. Also, note that $\varphi(h)=h\left(x_{\varphi}\right)=1$. Putting $\widetilde{f_{\gamma}}:=f_{\gamma} * h$ and $\widetilde{g_{\gamma}}=g_{\gamma} * h$, we have that $\widetilde{f}_{\gamma}$ and $\widetilde{g_{\gamma}}$ are contained in $C_{c}\left(U_{\gamma} \cap V_{\gamma}\right)$. Then it follows that $\widetilde{f}_{\gamma}^{*} * \widetilde{g_{\gamma}} \in C_{0}\left(G^{(0)}\right)$ and

$$
\begin{aligned}
\overline{\varphi\left(f_{\gamma}\right)} \varphi\left(g_{\gamma}\right) & =\overline{\varphi(h) \varphi\left(f_{\gamma}\right)} \varphi\left(g_{\gamma}\right) \varphi(h)=\varphi\left(\widetilde{f}_{\gamma}^{*} * \widetilde{g}_{\gamma}\right) \\
& ={\widetilde{f_{\gamma}}}^{*} * \widetilde{g_{\gamma}}\left(x_{\varphi}\right)=\overline{h(r(\gamma)) f_{\gamma}(\gamma)} g_{\gamma}(\gamma) h(s(\gamma))=1 .
\end{aligned}
$$

Now we have $\varphi\left(f_{\gamma}\right)=\varphi\left(g_{\gamma}\right)$ since $\varphi\left(f_{\gamma}\right) \in \mathbb{T}$.

Proposition 4.5. Let $G$ be an étale groupoid and $\varphi \in \Delta\left(C^{*}(G)\right)$. We define $\chi_{\varphi}: G_{x_{\varphi}} \rightarrow \mathbb{T}$ by $\chi_{\varphi}(\gamma):=\varphi\left(f_{\gamma}\right)$, where $\gamma \in G_{x_{\varphi}}$ and $f_{\gamma} \in C(G)$ is a function as in Proposition 4.4. Then $\chi_{\varphi}: G_{x_{\varphi}} \rightarrow \mathbb{T}$ is a group homomorphism.

Proof. Take $\alpha, \beta \in G_{x_{\varphi}}$. We show that $\chi_{\varphi}(\alpha) \chi_{\varphi}(\beta)=\chi_{\varphi}(\alpha \beta)$. Take $f_{\alpha}, f_{\beta} \in C(G)$ as in Proposition 4.4. It follows that $f_{\alpha} * f_{\beta} \in C_{c}(U)$ for some open bisection $U \subset G$ and $f_{\alpha} * f_{\beta}(\alpha \beta)=1$. Hence, we have

$$
\chi_{\varphi}(\alpha \beta)=\varphi\left(f_{\alpha} * f_{\beta}\right)=\varphi\left(f_{\alpha}\right) \varphi\left(f_{\beta}\right)=\chi_{\varphi}(\alpha) \chi_{\varphi}(\beta)
$$

by the definition of $\chi_{\varphi}$.

Proposition 4.6. Let $G$ be an étale groupoid. Then we have $\varphi=\varphi_{x_{\varphi}, \chi_{\varphi}}$ for all $\varphi \in$ $\Delta\left(C^{*}(G)\right)$.

Proof. Take $f \in C_{c}(U)$, where $U \subset G$ is an open bisection. It suffices to show that $\varphi(f)=\varphi_{x_{\varphi}, \chi_{\varphi}}(f)$, since $C^{*}(G)$ is generated by such functions. Note that $f^{*} * f \in$ $C_{c}\left(G^{(0)}\right)$. If $G_{x_{\varphi}} \cap f^{-1}(\mathbb{C} \backslash\{0\})=\emptyset$, then we have $0=f^{*} * f\left(x_{\varphi}\right)=|\varphi(f)|^{2}$. Since the restriction of $\left.f\right|_{G_{x_{\varphi}}}$ is zero, it follows that $\varphi_{x_{\varphi}, \chi_{\varphi}}(f)=0=\varphi(f)$. If $G_{x_{\varphi}} \cap f^{-1}(\mathbb{C} \backslash\{0\}) \neq$ $\emptyset, G_{x_{\varphi}} \cap f^{-1}(\mathbb{C} \backslash\{0\})$ is a singleton because $f$ is supported on an open bisection. Let $\gamma \in G_{x_{\varphi}} \cap f^{-1}(\mathbb{C} \backslash\{0\})$ be the unique element of $G_{x_{\varphi}} \cap f^{-1}(\mathbb{C} \backslash\{0\})$. Observe that $F:=f / f(\gamma) \in C_{c}(U)$ satisfies $F(\gamma)=1$. Now we have

$$
\varphi_{x_{\varphi}, \chi_{\varphi}}(f)=f(\gamma) \chi_{\varphi}(\gamma)=f(\gamma) \varphi(F)=\varphi(f) .
$$

Hence, $\varphi_{x_{\varphi}, \chi_{\varphi}}=\varphi$. 
Proposition 4.7. Let $G$ be an étale groupoid, $x \in G^{(0)}$ be a fixed point and $\chi: G_{x} \rightarrow \mathbb{T}$ be a group homomorphism. Then $x=x_{\varphi_{x, \chi}}$ and $\chi=\chi_{\varphi_{x, \chi}}$.

Proof. First, we show that $x=x_{\varphi_{x, \chi}}$. Take $f \in C_{c}\left(G^{(0)}\right)$ arbitrarily. Then we have

$$
f\left(x_{\varphi_{x, \chi}}\right)=\varphi_{x, \chi}(f)=f(x) \chi(x)=f(x) .
$$

Hence, it follows that $x=x_{\varphi_{x}}$.

Next, we show that $\chi=\chi_{\varphi_{x, \chi}}$. Take $\gamma \in G_{x}$ arbitrarily. There exist an open bisection $U \subset G$ with $\gamma \in U$ and $f \in C_{c}(U)$ with $f(\gamma)=1$. Then we have

$$
\chi_{\varphi_{x, \chi}}(\gamma)=\varphi_{x, \chi}(f)=f(\gamma) \chi(\gamma)=\chi(\gamma) .
$$

Hence, we have shown that $x=x_{\varphi_{x, \chi}}$ and $\chi=\chi_{\varphi_{x, \chi}}$.

Combining Propositions 4.6 and 4.7, we obtain the next theorem.

Theorem 4.8. Let $G$ be an étale groupoid. Define a set

$$
\begin{aligned}
& \mathcal{D}:=\left\{(x, \chi) \mid x \in G^{(0)}\right. \text { is a fixed point } \\
& \left.\qquad \text { and } \chi: G_{x} \rightarrow \mathbb{T} \text { is a group homomorphism }\right\} .
\end{aligned}
$$

Then the map

$$
\mathcal{D} \ni(x, \chi) \longrightarrow \varphi_{x, \chi} \in \Delta\left(C^{*}(G)\right)
$$

is bijective.

4.2. Construction of an étale abelian group bundle $\boldsymbol{G}^{\text {ab }}$. For an étale groupoid $G$, we construct an étale abelian group bundle $G^{\mathrm{ab}}$ so that $C^{*}(G)^{\mathrm{ab}} \simeq C^{*}\left(G^{\mathrm{ab}}\right)$ holds.

Proposition 4.9. Let $G$ be an étale group bundle. We define the commutator subgroupoid of $G$ by $[G, G]:=\bigcup_{x \in G^{(0)}}\left[G_{x}, G_{x}\right]$, where $\left[G_{x}, G_{x}\right]$ is the commutator subgroup of $G_{x}$. Then $[G, G]$ is an open normal subgroupoid of $G$.

Proof. It is obvious that $[G, G] \subset G$ is a normal subgroupoid. We show that $[G, G] \subset G$ is open. Take $\gamma \in[G, G]$. By the definition of the commutator subgroup, there exists $\left\{\alpha_{j}\right\}_{j=1}^{k},\left\{\beta_{j}\right\}_{j=1}^{k} \subset G_{s(\gamma)}$ such that

$$
\gamma=\alpha_{1} \beta_{1} \alpha_{1}^{-1} \beta_{1}^{-1} \alpha_{2} \beta_{2} \alpha_{2}^{-1} \beta_{2}^{-1} \cdots \alpha_{k} \beta_{k} \alpha_{k}^{-1} \beta_{k}^{-1} .
$$

Take open bisections $U_{j}, V_{j} \subset G$ such that $\alpha_{j} \in U_{j}$ and $\beta_{j} \in V_{j}$ for all $j=1,2, \ldots, k$. We show that $U_{1} V_{1} U_{1}^{-1} V_{1}^{-1} \subset[G, G]$, where we define $U^{-1}:=\left\{\gamma^{-1} \mid \gamma \in U\right\}$ for $U \subset G$. Fix $\gamma^{\prime} \in U_{1} V_{1} U_{1}^{-1} V_{1}^{-1}$. Then there exist $\alpha, \alpha^{\prime} \in U_{1}$ and $\beta, \beta^{\prime} \in V_{1}$ which satisfy $\gamma=$ $\alpha \beta \alpha^{\prime-1} \beta^{\prime-1}$. Since $G$ is a group bundle, we have $s(\alpha)=s\left(\alpha^{\prime}\right)=s(\beta)=s\left(\beta^{\prime}\right)$. We obtain $\alpha=\alpha^{\prime}$ and $\beta=\beta^{\prime}$ because $U_{1}$ and $V_{1}$ are bijections. Therefore, $\gamma^{\prime}=\alpha \beta \alpha^{-1} \beta^{-1} \in[G, G]$. Similarly, one can show that $U_{1} V_{1} U_{1}^{-1} V_{1}^{-1} U_{2} V_{2} U_{2}^{-1} V_{2}^{-1} \cdots U_{k} V_{k} U_{k}^{-1} V_{k}^{-1} \subset[G, G]$.

By Proposition 2.5, $U_{1} V_{1} U_{1}^{-1} V_{1}^{-1} U_{2} V_{2} U_{2}^{-1} V_{2}^{-1} \cdots U_{k} V_{k} U_{k}^{-1} V_{k}^{-1}$ is an open set and contains $\gamma$. Hence, $[G, G] \subset G$ is an open normal subgroupoid. 
Let $G$ be an étale groupoid. Recall that the set of all fixed points $F \subset G^{(0)}$ is a closed subset of $G^{(0)}$ (Proposition 2.7). We define $G_{\text {fix }}:=G_{F}$, which is an étale groupoid from Proposition 2.8. Since we have $G_{\mathrm{fix}}=\operatorname{Iso}\left(G_{\mathrm{fix}}\right), G_{\mathrm{fix}}$ is an étale group bundle.

Definition 4.10. Let $G$ be an étale groupoid. We define the abelianization of $G$ by $G^{\mathrm{ab}}:=G_{\mathrm{fix}} /\left[G_{\mathrm{fix}}, G_{\mathrm{fix}}\right]$.

Let $G$ be an étale groupoid. Then we have a *homomorphism $C^{*}(G) \rightarrow C^{*}\left(G_{\text {fix }}\right)$ induced by the restriction (Proposition 2.12). Composing with the *-homomorphism $C^{*}\left(G_{\mathrm{fix}}\right) \rightarrow C^{*}\left(G^{\mathrm{ab}}\right)$ in Proposition 3.13 , we obtain a ${ }^{*}$-homomorphism $\pi: C^{*}(G) \rightarrow$ $C^{*}\left(G^{\mathrm{ab}}\right)$.

Note that $C^{*}(G)$ is commutative if and only if $G$ is an étale abelian group bundle. In particular, $C^{*}\left(G^{\mathrm{ab}}\right)$ is commutative.

Lemma 4.11. Let $G$ be an étale groupoid. Then the map $\Phi: \Delta\left(C^{*}\left(G^{\mathrm{ab}}\right)\right) \ni \chi \mapsto \chi \circ \pi \in$ $\Delta\left(C^{*}(G)\right)$ is bijective.

Proof. Surjectivity of $\pi$ implies that $\Phi$ is injective. We show that $\Phi$ is surjective. Take $\varphi \in \Delta\left(C^{*}(G)\right)$. Then we have the fixed point $x_{\varphi} \in G^{(0)}$ and the group homomorphism $\chi_{\varphi}$ which makes the following diagram commutative:

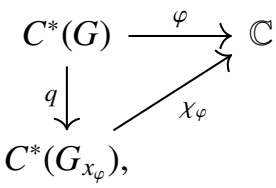

where $q: C^{*}(G) \rightarrow C^{*}\left(G_{x_{\varphi}}\right)$ is the *-homomorphism obtained in Proposition 2.12.

By the universality of $G_{x_{\varphi}}^{\mathrm{ab}}:=\left(G_{x_{\varphi}}\right)^{\mathrm{ab}}=\left(G^{\mathrm{ab}}\right)_{x_{\varphi}}$, we obtain the group homomorphism $\bar{\chi}_{\varphi}: G_{x_{\varphi}}^{\mathrm{ab}} \rightarrow \mathbb{T}$ that makes the following diagram commutative:

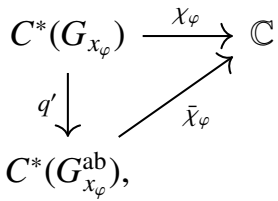

where $q^{\prime}: C^{*}\left(G_{x_{\varphi}}\right) \rightarrow C^{*}\left(G_{x_{\varphi}}^{\mathrm{ab}}\right)$ denotes the *-homomorphism induced by the quotient $\operatorname{map} G_{x_{\varphi}} \rightarrow G_{x_{\varphi}}^{\mathrm{ab}}$.

Let res: $C^{*}\left(G^{\mathrm{ab}}\right) \rightarrow C^{*}\left(G_{x_{\varphi}}^{\mathrm{ab}}\right)$ denote the *-homomorphism obtained by the restriction $C\left(G^{\mathrm{ab}}\right) \rightarrow C\left(G_{x_{\varphi}}^{\mathrm{ab}}\right)$ (see Proposition 2.12). Now we have the following commutative diagram: 


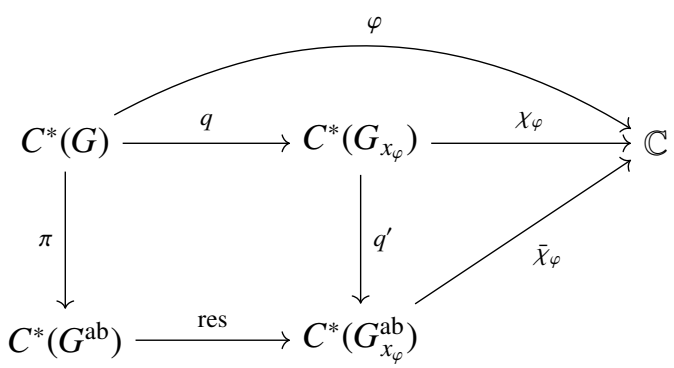

In particular, we have $\varphi=\left(\bar{\chi}_{\varphi} \circ\right.$ res $) \circ \pi$ and $\bar{\chi}_{\varphi} \circ$ res $\in \Delta\left(C^{*}\left(G^{\text {ab }}\right)\right)$. Hence, $\Phi$ is surjective.

We are now ready to calculate the abelianization of $C^{*}(G)$.

THEOREM 4.12. Let $G$ be an étale groupoid. Then $C^{*}(G)^{\mathrm{ab}}$ is isomorphic to $C^{*}\left(G^{\mathrm{ab}}\right)$ via the unique isomorphism $\bar{\pi}$ that makes the following diagram commutative:

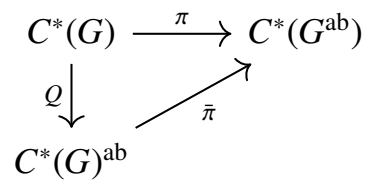

where $Q: C^{*}(G) \rightarrow C^{*}(G)^{\mathrm{ab}}$ denotes the quotient map.

Proof. By the universality of $C^{*}(G)^{\text {ab }}$, we obtain a *-homomorphism that makes the following diagram commutative;

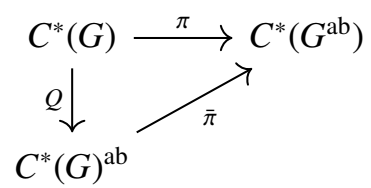

It is clear that $\bar{\pi}$ is surjective. We show that $\bar{\pi}$ is injective. Suppose that $a \in C^{*}(G)$ satisfies $\pi(a)=0$. It suffices to show $Q(a)=0$, which is equivalent to $\bar{\varphi}(Q(a))=0$ for all $\bar{\varphi} \in \Delta\left(C^{*}(G)^{\mathrm{ab}}\right)$ since $C^{*}(G)^{\mathrm{ab}}$ is commutative. Take $\bar{\varphi} \in \Delta\left(C^{*}(G)^{\mathrm{ab}}\right)$ and define $\varphi:=\bar{\varphi} \circ Q$. Then, by Lemma 4.11 , there exists $\tilde{\varphi} \in \Delta\left(C^{*}\left(G^{\mathrm{ab}}\right)\right)$ that makes the following diagram commutative;

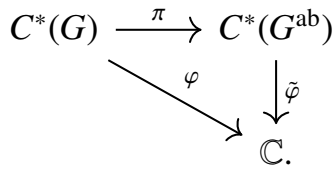

Now we have the following commutative diagram:

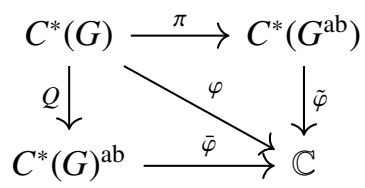


Hence, we have $\bar{\varphi}(Q(a))=\tilde{\varphi}(\pi(a))=0$.

4.3. Duals of étale abelian group bundles. Let $G$ be an étale groupoid. Since the abelianization of $C^{*}(G)$ is a commutative $C^{*}$-algebra, $C^{*}(G)^{\text {ab }}$ is isomorphic to $C_{0}\left(\Delta\left(C^{*}(G)^{\mathrm{ab}}\right)\right.$ ) via the Gelfand transformation (see, for example, [2, Theorem II.2.2.4]). In this subsection we calculate the Gelfand spectrum $\Delta\left(C^{*}(G)^{\mathrm{ab}}\right)$.

For a discrete abelian group $\Gamma$, its Pontryagin dual group is defined as the set of all group homomorphisms from $\Gamma$ to $\mathbb{T}$, which is denoted by $\widehat{\Gamma}$. Then $\widehat{\Gamma}$ is an abelian group with respect to pointwise multiplication. It is known that $\widehat{\Gamma}$ is a compact abelian topological group with respect to the topology of pointwise convergence.

Proposition 4.13. Let $\Gamma$ be a discrete group and let $Q: C^{*}(\Gamma) \rightarrow C^{*}\left(\Gamma^{\mathrm{ab}}\right)$ be the *-homomorphism induced by the quotient map $\Gamma \rightarrow \Gamma^{\mathrm{ab}}$. Then the map

$$
\Phi: \widehat{\Gamma^{\mathrm{ab}}} \ni \chi \mapsto \chi \circ Q \in \Delta\left(C^{*}(\Gamma)\right)
$$

is a homeomorphism. Hence, $C^{*}(\Gamma)^{\mathrm{ab}}$ is isomorphic to $C\left(\widehat{\Gamma^{\mathrm{ab}}}\right)$.

Proof. This follows from the universality of $\Gamma^{\mathrm{ab}}$ and $C^{*}(\Gamma)$.

As seen in the previous proposition, the key to calculating $\Delta\left(C^{*}(G)\right)$ is the Pontryagin dual.

Definition 4.14. Let $G$ be an étale abelian group bundle. We define a group bundle $\widehat{G}:=\left\{(\chi, x) \mid x \in G^{(0)}, \chi \in \widehat{G_{x}}\right\}$ over $G^{(0)}$.

Note that $\widehat{G}$ is a group bundle such that $\widehat{G_{x}}=\widehat{G_{x}} \times\{x\}\left(\simeq \widehat{G_{x}}\right)$ for every $x \in G^{(0)}$.

Let $G$ be an étale abelian group bundle and $(\chi, x) \in \widehat{G}$. Recall that we obtain the *-homomorphism $\varphi_{x, \chi} \in \Delta\left(C^{*}(G)\right)$ as in Definition 4.1.

Definition 4.15. Let $G$ be an étale abelian group bundle. For each $f \in C(G)$, we define $\operatorname{ev}_{f}: \widehat{G} \rightarrow \mathbb{C}$ by $\operatorname{ev}_{f}((\chi, x))=\varphi_{x, \chi}(f)$, where $(\chi, x) \in \widehat{G}$. We define a topology of $\widehat{G}$ as the weakest topology in which $\mathrm{ev}_{f}$ is continuous for all $f \in C(G)$.

Proposition 4.16. Let $G$ be an étale abelian group bundle. Then the map

$$
\Psi: \Delta\left(C^{*}(G)\right) \ni \varphi \mapsto\left(\chi_{\varphi}, x_{\varphi}\right) \in \widehat{G}
$$

is a homeomorphism (see Propositions 4.2 and 4.5 for the definition of $x_{\varphi}$ and $\chi_{\varphi}$ ). Hence, $C^{*}(G)$ is isomorphic to $C_{0}(\widehat{G})$.

Proof. Proposition 4.8 states that $\Psi$ is a bijection and $\Psi^{-1}$ is given by $\Psi^{-1}((\chi, x))=\varphi_{x, \chi}$ for each $(\chi, x) \in \widehat{G}$. For each $f \in C(G)$, a map $\Delta\left(C^{*}(G)\right) \ni \varphi \mapsto \operatorname{ev}_{f}\left(\left(\chi_{\varphi}, x_{\varphi}\right)\right)=\varphi(f) \in \mathbb{C}$ is continuous. This means that $\Psi$ is continuous. The continuity of $\Psi^{-1}$ follows from approximation arguments. Therefore, $\Psi$ is a homeomorphism.

Let $G$ be an étale groupoid. Recall that $G^{\mathrm{ab}}$ is an étale abelian group bundle.

Corollary 4.17. Let $G$ be an étale groupoid. Then $C^{*}(G)^{\mathrm{ab}}$ is isomorphic to $C_{0}\left(\widehat{G^{\mathrm{ab}}}\right)$. 
Proof. Recall that $C^{*}(G)^{\mathrm{ab}}$ is isomorphic to $C^{*}\left(G^{\mathrm{ab}}\right)$ by Theorem 4.12. Since $G^{\mathrm{ab}}$ is an étale abelian group bundle, Proposition 4.16 implies that $C^{*}\left(G^{\mathrm{ab}}\right)$ is isomorphic to $C_{0}\left(\widehat{G^{\mathrm{ab}}}\right)$.

Proposition 4.18. Let $G$ be an étale abelian group bundle. Then $\widehat{G}$ is a locally compact Hausdorff topological group bundle. Furthermore, $\widehat{G}$ is compact if and only if $G^{(0)}$ is compact.

Proof. It is clear that $\widehat{G}$ is locally compact Hausdorff, since $\widehat{G}$ is homeomorphic to $\Delta\left(C^{*}(G)\right)$. In order to show the continuity of the operations, take $f \in C(G)$ arbitrarily. Then the map $\widehat{G}^{(2)} \ni\left(\chi_{1}, \chi_{2}\right) \mapsto \operatorname{ev}_{f}\left(\chi_{1} \chi_{2}\right)=\operatorname{ev}_{f}\left(\chi_{1}\right) \operatorname{ev}_{f}\left(\chi_{2}\right) \in \mathbb{C}$ is continuous. Therefore, the multiplication of $\widehat{G}^{(2)}$ is continuous. Similarly, one can show that the inverse is continuous. Hence, $\widehat{G}$ is a locally compact Hausdorff topological group bundle. The last assertion follows from the fact that $G^{(0)}$ is compact if and only if $C^{*}(G) \simeq C_{0}(\widehat{G})$ is unital.

Example 4.19. We give an example of an étale groupoid $G$ such that $G^{\text {ab }}$ is not Hausdorff although $G$ is Hausdorff. Let $\Xi_{3}=\langle s, t| s^{3}=t^{2}=e$, st $\left.=t s^{2}\right\rangle=$ $\left\{e, s, s^{2}, t, t s, t s^{2}\right\}$ be the symmetric group of degree 3 and $A_{3}:=\left\{e, s, s^{2}\right\} \subset \Im_{3}$ be the subgroup of even permutations. Let $G:=\Xi_{3} \times[0,1] \backslash\left\{(t, 1) \mid t \notin A_{3}\right\}$ be an étale group bundle over [0,1]. Then $G$ can be drawn as follows:

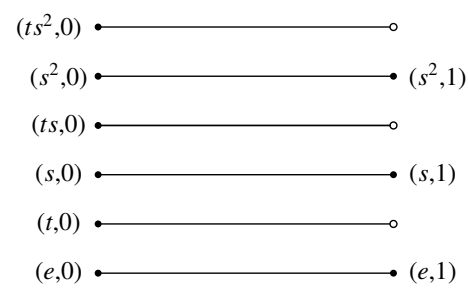

One can see that $[G, G] \subset G$ is not closed. By Proposition 3.11, $G^{\mathrm{ab}}=G /[G, G]$ is not Hausdorff. Indeed, letting $q: G \rightarrow G^{\text {ab }}$ denote the quotient map, $G^{\text {ab }}$ looks as follows:

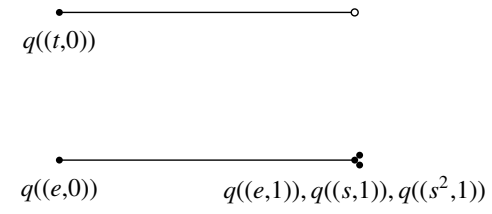

The dual $\widehat{G^{\mathrm{ab}}}$ of $G^{\mathrm{ab}}$ can be drawn as follows:

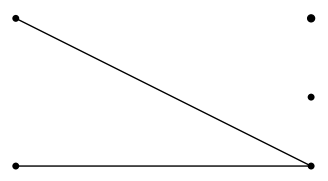

Note that $\widehat{G^{\mathrm{ab}}}$ is not étale. 


\section{Acknowledgements}

The author would like to thank his supervisor, Professor Takeshi Katsura, for fruitful discussions and guidance. The author is also grateful to the referees for useful comments.

\section{References}

[1] B. Blackadar, 'Shape theory for C*-algebras', Math. Scand. 56 (1985), 249-275.

[2] B. Blackadar, Operator Algebras: Theory of $C^{*}$-Algebras and von Neumann Algebras, Encyclopaedia of Mathematical Sciences (Springer, Berlin, 2006).

[3] J. Brown, L. O. Clark, C. Farthing and A. Sims, 'Simplicity of algebras associated to étale groupoids', Semigroup Forum (2) 88 (2014), 433-452.

[4] L. O. Clark, R. Exel, E. Pardo, A. Sims and C. Starling, 'Simplicity of algebras associated to non-Hausdorff groupoids', Trans. Amer. Math. Soc. (5) 372 (2019), 3669-3712.

[5] A. Connes, 'A survey of foliations and operator algebras', Proc. Sympos. Pure Math. 38(1) (1982), 521-628.

[6] R. Exel, 'Inverse semigroups and combinatorial C*-algebras', Bull. Brazilian Math. Soc., New Series 39 (2007), 191-313.

[7] R. Exel, 'Non-Hausdorff étale groupoids', Proc. Amer. Math. Soc. (3) 139 (2011), 897-907.

[8] R. Exel and E. Pardo, 'The tight groupoid of an inverse semigroup', Semigroup Forum (1) 92 (2016), 274-303.

[9] A. Paterson, Groupoids, Inverse Semigroups, and their Operator Algebras, Progress in Mathematics (Birkhäuser, Boston, 2012).

[10] J. Renault, A Groupoid Approach to $C^{*}$-Algebras, Lecture Notes in Mathematics (Springer, Berlin, 1980).

[11] J. Renault, 'Cartan subalgebras in C*-algebras', Irish Math. Soc. Bull. 61 (2008), 29-63.

[12] A. Sims, 'Hausdorff étale groupoids and their $\mathrm{C}^{*}$-algebras', in: Operator Algebras and Dynamics: Groupoids, Crossed Products and Rokhlin Dimension, Advanced Courses in Mathematics (ed. F. Perera) (CRM Barcelona, Birkhäuser, 2020).

FUYUTA KOMURA, Department of Mathematics,

Faculty of Science and Technology, Keio University, 3-14-1 Hiyoshi,

Kohoku-ku, Yokohama 223-8522, Japan

e-mail: fuyuta.k@keio.jp

and

Mathematical Science Team, Center for Advanced Intelligence Project (AIP),

RIKEN, Nihonbashi 1-chome Mitsui Building, 15th Floor,

1-4-1 Nihonbashi, Chuo-ku, Tokyo 103-0027, Japan 